\title{
Associação entre insatisfação com a imagem corporal e indicadores antropométricos em adolescentes
}

CDD. 20.ed. 302.222

796.033

\author{
Maria Fátima GLANER* \\ Andreia PELEGRINI** \\ Claudio Olavo CORDOBA*** \\ Maria Elizete POZZOBON ${ }^{* * * * *}$
}

*Centro de Educação
Física e Esportes,
Universidade Estadual
de Londrina.
${ }^{* *}$ Centro de Ciências
Humanas, Educação
e Letras, Universidade
Estadual do Oeste do
Paraná.
*** Un i ve r si da de
Católica de Brasília.
****Centro de Ciências
da Saúde, Universidade
Comunitária da Região
de Chapecó.

0 objetivo foi verificar a associação entre a insatisfação com a imagem corporal, o índice de massa corpora anos) de ambos os sexos. Foram medidos massa corporal, estatura, perímetro do abdômen, dobras cutâneas do tríceps e perna medial e, coletadas informações referentes a imagem corporal. O IMC, a obesidade abdominal e a adiposidade corporal foram usados como referência em relação ao desfecho. A prevalência de insatisfação com a imagem corporal foi de $60 \%$ (rapazes $=54,3 \%$, moças $=65,2 \% ; p<0,05$ ). Os rapazes com IMC baixo e obesidade abdominal apresentaram, respectivamente, 4,31 e 4,93 vezes mais chance de insatisfação corporal. As moças com IMC alto e adiposidade corporal alta apresentaram, respectivamente, 6,81 e 1,95 vezes mais chance de insatisfação corporal. Enquanto nos rapazes o IMC baixo e a obesidade abdominal apresentaram associação com a insatisfação corporal; nas moças o IMC e a adiposidade corporal elevados estiveram associados.

PalAVRAS-ChaVE: Antropometria; Imagem corporal; Dobras cutâneas; Gordura abdominal; IMC.

\section{Introdução}

Os padrões de beleza impostos pela mídia e sociedade preconizam uma forma de corpo esbelto para as moças e atlético para os rapazes ${ }^{1}$. Em consequência, o perfil antropométrico em voga tem provocado elevados níveis de descontentamento com o próprio corpo $^{2}$ e, tem gerado um aumento na insatisfação com a imagem corporal em adolescentes ${ }^{3}$. Estudos conduzidos em diferentes locais do Brasil apontam prevalências elevadas de insatisfação com a imagem corporal em adolescentes ${ }^{4-6}$.

Muitas vezes o biotipo não está associado à saúde fisiológica, apesar de o sujeito estar satisfeito com a sua imagem corporal. Desse modo, indicadores antropométricos de gordura corporal total, ou localizada, servem de parâmetro para indicar se a insatisfação com a imagem corporal é consequência do baixo ou excessivo acúmulo de gordura. Além disso, estudos apontam que a baixa quantidade de gordura $^{7-8}$ e seu acúmulo excessivo e/ou localizado ${ }^{9-11}$ está associado a diversas doenças comportamentais e crônicas degenerativas não transmissíveis, respectivamente. Nesse sentido, o presente estudo tem como objetivo verificar a associação entre a insatisfação com a imagem corporal, índice de massa corporal, adiposidade corporal e obesidade abdominal em adolescentes.

\section{Método}

O estudo sobre a associação entre a insatisfação com a imagem corporal e os indicadores antropométricos em adolescentes foi desenvolvido a partir de um estudo transversal intitulado "Interação entre variáveis que podem influenciar no acúmulo de gordura corporal e no perfil lipídico de pais e filhos", 
aprovado por um comitê de ética institucional (Parecer 026/2009). O presente estudo foi realizado em uma amostra representativa de adolescentes do município de Saudades - SC, localizado no extremo Oeste do estado de Santa Catarina, região Sul do Brasil. Esse município é predominantemente formado por descendentes de alemães e caracterizase por ser de pequeno porte, constituído por 8.880 habitantes ${ }^{12}$. Apresenta o Índice de Desenvolvimento Humano de $0,82^{13}$, sendo classificado com alto desenvolvimento humano.

A população do estudo foi composta por 1.381 adolescentes com idade de 11 a 17 anos $^{12}$, matriculados em duas escolas públicas. Em Saudades - SC não há escolas privadas, neste sentido, selecionou-se, intencionalmente, a única escola que atende alunos do $5^{\circ}$ ano do ensino fundamental até o $3^{\circ}$ ano do ensino médio, localizada na sede do município. Outra, localizada na área rural, que atende somente alunos do ensino fundamental. Os alunos, da respectiva faixa etária envolvida no estudo, foram convidados em sala de aula e por meio de uma "carta convite" que levaram aos pais ou responsáveis legais.

Para a determinação do tamanho da amostra, foram utilizados os procedimentos sugeridos por LUIZ e MAGNANINI ${ }^{14}$, a partir de uma população finita, considerando-se a prevalência de insatisfação com a imagem corporal de $64 \%{ }^{15}$, intervalo de confiança 95\% (IC95\%), erro estimado de cinco pontos percentuais e acréscimo de $20 \%$ como possível índice de perda. Assim, estimou-se que seria necessário coletar informações de 541 adolescentes. Em virtude das características do processo de amostragem que envolveu todos os indivíduos pertencentes aos conglomerados (escolas), participaram da amostra 708 adolescentes. Foram excluídos da amostra os adolescentes com idade superior a 17 anos $(n=42)$ e se considerou como perda amostral os adolescentes que não realizaram as medidas de massa corporal e estatura $(n=60)$, perímetro do abdômen $(n=40)$, dobras cutâneas $(n=40)$ e não responderam o questionário sobre percepção da imagem corporal $(\mathrm{n}=$ 40). Desta forma, a amostra final foi composta por 637 adolescentes, com idade de 11 a 17 anos, de ambos os sexos, domiciliados nas áreas urbana e rural.
As informações da percepção da imagem corporal foram obtidas com a utilização da escala de nove silhuetas corporais proposta por STUNKARD et al. ${ }^{16}$. O conjunto de silhuetas era mostrado aos adolescentes, e, os mesmos respondiam a duas perguntas: Qual a silhueta que melhor representa a sua aparência corporal atual (real)? Qual é a silhueta corporal que você gostaria de ter (ideal)? Quando a variação entre a silhueta real e a ideal foi igual a zero, os adolescentes foram classificados como satisfeitos; e se diferente de zero, insatisfeitos.

As variáveis antropométricas (massa corporal $\mathrm{MC}$, estatura - ES, perímetro do abdômen - PAB e espessura de dobras cutâneas das regiōes do tríceps e perna medial) foram mensuradas seguindo procedimentos descritos em Petroski ${ }^{17}$. Os indicadores antropométricos analisados foram: a) índice de massa corporal $\left(\mathrm{IMC}=\mathrm{MC}_{\mathrm{kg}} / \mathrm{ES}_{\mathrm{m}}^{2}\right)$; b) adiposidade corporal por meio do somatório de espessura de duas dobras cutâneas ( $\left.\sum 2 \mathrm{DC}\right)$; e, c) obesidade abdominal por meio do perímetro do abdômen.

Para a classificação do IMC utilizou-se os pontos de corte para adolescentes, considerando a idade e o sexo ${ }^{18-19}$ : baixo $\left(\mathrm{IMC}<18,5 \mathrm{~kg} / \mathrm{m}^{2}\right)$, ideal (IMC entre 18,5 e $\left.25 \mathrm{~kg} / \mathrm{m}^{2}\right)$ e alto $\left(\mathrm{IMC}>25 \mathrm{~kg} / \mathrm{m}^{2}\right)$. A adiposidade corporal foi classificada segundo a AAHPERD $^{20}$ : baixa $\left(\sum 2 \mathrm{DC}<16 \mathrm{~mm}\right.$ ou $\left.<12 \mathrm{~mm}\right)$, ideal ( $\sum 2 \mathrm{DC}$ entre 16 e $36 \mathrm{~mm}$ ou entre 12 e $25 \mathrm{~mm}$ ) e alta $\left(\sum 2 \mathrm{DC}>36 \mathrm{~mm}\right.$ ou $\left.>25 \mathrm{~mm}\right)$, para moças e rapazes, respectivamente. A obesidade abdominal foi classificada segundo os pontos de corte de FERNANDEZ et al. ${ }^{21}$ : ideal ( $\leq$ percentil 75$)$ e alto (> percentil 75 ).

$\mathrm{Na}$ análise descritiva das variáveis foram utilizadas médias, desvios padrão e distribuição de frequências. Utilizou-se o teste " $\mathrm{t}$ " de Student para amostras independentes e o qui-quadrado para comparar os valores absolutos e relativos, respectivamente. Nas comparaçōes entre duas proporções foi aplicado o teste de significância para diferenças entre proporções. A associação entre a insatisfação com a imagem corporal e os indicadores antropométricos foi analisada por regressão logística. Foram testados dois modelos, um simples e outro múltiplo. Em todas as análises foi fixado o nível de significância de 5\%. A análise estatística foi realizada utilizando o SPSS, versão 15.0 e o MedCalc versão 9.3.3.0. 


\section{Resultados}

As características gerais da amostra estão apresentadas na TABELA 1 . Diferenças $(\mathrm{p}<0,05)$ entre os sexos foram encontradas nas médias da MC, ES, $\sum 2 \mathrm{DC}$, silhueta atual e silhueta ideal. Em todas as variáveis os rapazes apresentaram valores superiores às moças, exceto no $22 \mathrm{DC}$.

TABELA 1 - Características gerais da amostra. Saudades, Santa Catarina, 2008.

\begin{tabular}{lccc}
\hline Variáveis & Rapazes $(\mathbf{n}=\mathbf{3 0 4})$ & Moças $(\mathbf{n}=\mathbf{3 3 3})$ & Total $(\mathbf{n}=\mathbf{6 3 7})$ \\
\hline Idade $(\mathrm{anos})$ & $14,4 \pm 1,8$ & $14,2 \pm 1,9$ & $14,3 \pm 1,9$ \\
$\mathrm{MC}(\mathrm{kg})^{*}$ & $55,6 \pm 13,6$ & $51,5 \pm 10,8$ & $53,5 \pm 12,3$ \\
$\mathrm{ES}(\mathrm{cm})^{*}$ & $164,9 \pm 11,6$ & $158,9 \pm 8,6$ & $161,8 \pm 10,6$ \\
$\mathrm{IMC}\left(\mathrm{kg} / \mathrm{m}^{2}\right)$ & $20,2 \pm 3,2$ & $20,2 \pm 3,2$ & $20,2 \pm 3,2$ \\
PAB $(\mathrm{cm})^{*}$ & $72,4 \pm 8,6$ & $70,5 \pm 8,0$ & $71,4 \pm 8,3$ \\
$\sum 2 \mathrm{DC}(\mathrm{mm})^{*}$ & $29,0 \pm 12,9$ & $40,1 \pm 12,8$ & $34,8 \pm 14,9$ \\
Silhueta atual & $4,3 \pm 1,2$ & $4,0 \pm 1,0$ & $4,1 \pm 1,1$ \\
Silhueta ideal $^{*}$ & $4,2 \pm 0,8$ & $3,4 \pm 0,8$ & $3,8 \pm 0,9$ \\
\hline
\end{tabular}

${ }^{*} \mathrm{MC}=$ massa corporal; $\mathrm{ES}$ = estatura; IMC = índice de massa corporal;

$\mathrm{PAB}=$ perímetro do abdômen;

$\Sigma 2 \mathrm{DC}=$ somatório de espessura de duas dobras cutâneas.

${ }^{*} p<0,05$ para diferença entre os sexos (teste "t" de Student para amostras independentes).
A TABELA 2 mostra a proporção de adolescentes, por sexo, de acordo com os indicadores antropométricos (IMC, $\sum 2 \mathrm{DC}$ e obesidade abdominal). Não houve associação $(p>0,05)$ entre os indicadores antropométricos e o sexo. Verifica-se que $77,2 \%$ dos adolescentes estão na classificação ideal para o IMC, ao passo que, no $\sum 2 \mathrm{DC}$, apenas 42,7\% estão nessa condição. Enquanto 7,5\% dos adolescentes são classificados com baixo IMC, $2 \%$ são classificados com baixo $\sum 2 \mathrm{DC}$. Observou-se ainda que $55,3 \%$ dos adolescentes apresentam adiposidade corporal alta pela classificação no $\sum 2 \mathrm{DC}$, e apenas 15,2\% são classificados com IMC alto.

TABELA 2 - Distribuição dos adolescentes, por sexo, de acordo com os indicadores antropométricos. Saudades, Santa Catarina, 2008.

\begin{tabular}{|c|c|c|c|c|}
\hline \multirow{2}{*}{$\begin{array}{l}\text { Indicadores } \\
\text { antropométricos }\end{array}$} & Total & Rapazes & Moças & \multirow[b]{2}{*}{$\mathbf{p}$} \\
\hline & n (\%) & n (\%) & n (\%) & \\
\hline IMC & & & & 0,488 \\
\hline Ideal & $492(77,2)^{*}$ & $237(78,0)$ & $255(76,6)$ & \\
\hline Baixo & $48(7,5)^{*}$ & $19(6,3)$ & $29(8,7)$ & \\
\hline Alto & $97(15,2)^{*}$ & $48(15,8)$ & $49(14,7)$ & \\
\hline Obesidade abdominal & & & & 0,570 \\
\hline Sem & $552(86,7)$ & $261(85,9)$ & $291(87,4)$ & \\
\hline Com & $85(13,3)$ & $43(14,1)$ & $42(12,6)$ & \\
\hline$\sum 2 \mathrm{DC}$ & & & & 0,106 \\
\hline Ideal & $272(42,7)^{*}$ & $143(47)$ & $129(38,7)$ & \\
\hline Baixo & $13(2,0)^{*}$ & $6(2,0)$ & $7(2,1)$ & \\
\hline Alto & $352(55,3)^{*}$ & $155(51,0)$ & $197(59,2)$ & \\
\hline
\end{tabular}

IMC = índice de massa corporal;

$\sum 2 \mathrm{DC}=$ somatório de espessura de duas dobras cutâneas.

* Comparação entre IMC e $\Sigma 2 \mathrm{DC}$ (teste para comparação entre as proporções).
A prevalência de insatisfação com a imagem corporal entre os adolescentes do presente estudo foi de $60 \%$, sendo maior nas moças $(65,2 \%)$ do que nos rapazes $(54,3 \%$; $\mathrm{p}<0,05)$. Para os rapazes (TABELA 3 ) as análises univariadas indicam, no modelo simples, associação do IMC e da obesidade abdominal com a insatisfação com a imagem corporal. No modelo ajustado para todas as variáveis, o IMC e a obesidade abdominal permaneceram associados, revelando que os rapazes com baixo IMC apresentaram 4,31 (IC95\% = 1,37-13,53) vezes mais chance de insatisfação do que aqueles com IMC ideal. Além disso, os 
$\mathrm{OR}=$ odds ratio;

$I C=$ intervalo de confiança;

IMC = índice de massa corporal;

$\Sigma 2 \mathrm{DC}=$ somatório de espessura de duas dobras cutâneas.

$\mathrm{OR}^{* *}=$ odds ratio ajustada para todas as variáveis. rapazes com obesidade abdominal têm 4,93 (IC95\% $=1,57-15,54)$ vezes mais chance de insatisfação com a imagem corporal que os sem obesidade abdominal.

Nas moças, os resultados do modelo simples revelaram associação do desfecho com todos os indicadores antropométricos (TABELA 3). Quando o modelo foi ajustado para todas as variáveis, o IMC e o $\sum 2 \mathrm{DC}$ permaneceram associados. As moças com IMC alto apresentaram 6,81 $($ IC95\% $=1,69-27,48)$ vezes mais chance de insatisfação que as com IMC ideal. Aquelas com classificação alta no $\sum 2 \mathrm{DC}$ apresentaram $1,95(\mathrm{IC} 95 \%=1,17-3,25)$ vezes mais chance de insatisfação com a imagem corporal do que aquelas com $\sum 2 \mathrm{DC}$ ideal.

TABELA 3 - Associação da insatisfação com a imagem corporal com os indicadores antropométricos - regressão logística (categoria de referência: satisfeito com a imagem corporal). Saudades, Santa Catarina, 2008.

\begin{tabular}{|c|c|c|c|c|}
\hline \multirow{2}{*}{$\begin{array}{l}\text { Indicadores } \\
\text { antropométricos }\end{array}$} & \multirow{2}{*}{\multicolumn{2}{|c|}{$\begin{array}{c}\text { Rapazes } \\
R^{* *}(\operatorname{IC} 05 \%)\end{array}$}} & Moças & \multirow[b]{2}{*}{$\mathrm{OR}^{* *}(\mathrm{IC} 95 \%)$} \\
\hline & & & OR (IC95\%) & \\
\hline \multicolumn{5}{|l|}{ IMC } \\
\hline Ideal & 1 & 1 & 1 & 1 \\
\hline Baixo & $4,19(1,35-12,98)$ & $4,31(1,37-13,53)$ & $0,79(0,37-1,72)$ & $1,11(0,47-2,60)$ \\
\hline Alto & $4,24(2,02-8,91)$ & $1,54(0,56-4,24)$ & $9,89(3,00-32,67)$ & $6,81(1,69-27,5)$ \\
\hline \multicolumn{5}{|l|}{ Obesidade abdominal } \\
\hline Sem & 1 & 1 & 1 & 1 \\
\hline Com & $6,41(2,62-15,70)$ & $4,93(1,57-15,54)$ & $4,56(1,74-11,96)$ & $1,14(0,34-3,79)$ \\
\hline \multicolumn{5}{|l|}{$\sum 2 \mathrm{DC}$} \\
\hline Ideal & 1 & 1 & 1 & 1 \\
\hline Baixo & $2,15(0,38-12,08)$ & $1,65(0,27-10,03)$ & $1,23(0,27-5,74)$ & $1,17(0,24-5,82)$ \\
\hline Alto & $1,57(0,99-2,48)$ & $1,09(0,65-1,82)$ & $2,65(1,66-4,24)$ & $1,95(1,17-3,25)$ \\
\hline
\end{tabular}

\section{Discussão}

Mais da metade (60\%) dos adolescentes investigados na presente casuística está insatisfeita com a imagem corporal. Prevalências similares foram encontradas em estudos conduzidos em adolescentes domiciliados na área rural e urbana de pequenos municípios $^{5}$ e em grandes centros urbanos ${ }^{6,22-23}$. Prevalências mais elevadas foram encontradas em adolescentes residentes na área urbana de um município de pequeno porte ${ }^{4}$. Dado as características da amostra, não era esperado encontrar prevalência tão elevada de insatisfação com a imagem corporal. Parece, então, que os fatores que levam os adolescentes de centros urbanos ao descontentamento com seu corpo, já afetam aqueles domiciliados em áreas rurais e em pequenas cidades.

As proporções elevadas de insatisfação com a imagem corporal na adolescência podem ser explicadas pelos avanços tecnológicos, juntamente com os meios de comunicação que influenciam os padrões de beleza na atualidade, sugerindo como ideal um corpo magro, esguio ou atlético ${ }^{24}$. Todavia, estes padrões nem sempre consideram os aspectos da saúde e as diferentes constituições físicas da população ${ }^{25}$, o que acarreta, muitas vezes, em uma imagem corporal negativa, representada por altos níveis de insatisfação com a percepção do $\operatorname{corpo}^{26}$. Cabe ressaltar que o presente estudo não analisou o impacto das transformaçóes tecnológicas e dos meios de comunicação na percepção da imagem corporal. Por outro lado, a literatura científica é vasta em apontar que, o aumento da prevalência do sobrepeso e da obesidade está, também, relacionado ao avanço tecnológico. Assim, a insatisfação com a imagem corporal pode não estar associada diretamente ao avanço tecnológico, e sim com as mazelas decorrentes ou influenciadas por este.

Foi observada maior prevalência de insatisfação corporal nas moças $(65,2 \%)$ do que nos rapazes $(54,3 \%)$. Esses resultados corroboram os estudos nacionais ${ }^{15,27}$, entretanto, divergem dos achados obtidos por Pelegrini e Petroski ${ }^{6}$, os quais encontraram prevalência de insatisfação corporal mais 
elevada no sexo masculino $(72,6 \%)$ em relação ao feminino $(61,8 \%)$. Outras investigaçōes ${ }^{5,23}$ revelaram similaridade na insatisfação com a imagem corporal entre os sexos.

Um achado interessante encontrado no presente estudo foi a diferença significativa entre os indicadores antropométricos IMC e $\Sigma 2$ DC. Enquanto a maioria dos adolescentes foi classificada com IMC ideal, o $\sum 2 \mathrm{DC}$ indicou que mais da metade destes se encontravam com alta adiposidade corporal (TABELA 2). GLANER ${ }^{28}$ ao conduzir uma pesquisa em adolescentes, verificou que apenas $49 \%$ das moças e $57 \%$ dos rapazes foram classificados concomitantemente pelo IMC e $\Sigma 2 \mathrm{DC}$. Dado o fato de que a maioria apresentou insatisfação com a imagem corporal e, que mais da metade da amostra apresentou alto $\sum 2 \mathrm{DC}$, este pode ser um melhor indicador antropométrico de insatisfação com a imagem corporal do que o IMC. Além do fato que o $\sum 2 \mathrm{DC}$ é um estimador da adiposidade corporal, enquanto que o IMC indica somente a quantidade de massa por superfície.

Nas análises univariadas (TABELA 3), foi observada associação entre a insatisfação com a imagem corporal e os indicadores antropométricos. No sexo masculino, o IMC e a obesidade abdominal estiveram associados à insatisfação com a imagem corporal, indicando que àqueles com baixo IMC e com obesidade abdominal apresentaram aproximadamente cinco vezes mais chance de insatisfação com a imagem corporal. Os resultados encontrados na associação do IMC baixo com a insatisfação com a imagem corporal corroboram os achados de PETROSKI et al. ${ }^{5}$, e divergem de outras pesquisas ${ }^{22-23,29}$. Baixos valores de IMC, necessariamente, não estão associados a fatores de risco à saúde no que concerne às doenças crônicas não transmissíveis (ex.: cardiovasculares, diabetes tipo II). No entanto, podem estar associados à bulimia, anorexia ou desnutrição calórica proteica. Ao passo que, a obesidade abdominal está diretamente associada a vários fatores de risco de doenças cardiovasculares, os quais começam a se instalar silenciosamente durante a infância e a adolescência. $\mathrm{O}$ fato do baixo IMC se associar com a insatisfação com a imagem corporal reforça que os rapazes desejam um porte atlético ${ }^{1}$.

Nas moças, foi observada associação entre insatisfação com a imagem corporal com o IMC e o $\Sigma 2 D C$. Estes resultados indicaram que moças com o IMC e o $\sum 2 \mathrm{DC}$ alto apresentaram, respectivamente, 6,8 e 1,95 vezes mais chance de insatisfação com a imagem corporal quando comparadas àquelas com o IMC e o $\sum 2 \mathrm{DC}$ ideal. Estes resultados corroboram os dados encontrados na literatura, os quais revelam que as moças com adiposidade corporal elevada tendem a apresentar maior insatisfação com a imagem corporal $^{4-5,22-23,29}$. A associação do IMC e $\sum 2$ DC alto com a insatisfação com a imagem corporal reforça que as moças almejam um corpo esbelto ${ }^{1}$.

Apensar, pesquisas nacionais e internacionais ${ }^{5,22-23,29}$ destacam que a insatisfação corporal se mostra mais prevalente em crianças e adolescentes que apresentam o IMC alto. Evidências apontam que, mesmo entre aqueles sem excesso de peso há associação entre o IMC e a insatisfação com a imagem corporal $^{30}$.

As proporções elevadas de insatisfação com a imagem corporal em adolescentes são preocupantes. Diversos problemas e distúrbios estão associados com a imagem corporal negativa, tais como a anorexia e a bulimia ${ }^{7-8}$, a baixa autoestima ${ }^{31}$ e a depressão ${ }^{32}$. Complementando, o sobrepeso e a obesidade têm aumentado sensivelmente em crianças e adolescentes ${ }^{33-34}$, estando associados a fatores de risco cardiovascular ${ }^{9-10}$.

Por um lado, a maioria das evidências direcionam para o fato dos adolescentes estarem mais preocupados em atingir um físico ideal, somente com fins de satisfação pessoal, deixando de lado a saúde fisiológica, por outro, em recente publicação com a mesma população do presente estudo, Petroski et al. ${ }^{35}$ evidenciaram que a saúde $(95,5 \%)$ e a estética $(96,8 \%)$ são os motivos que mais influenciam a insatisfação com a imagem corporal. Nesse sentido, as ações destinadas à promoção da saúde fisiológica também podem repercutir positivamente sobre a satisfação com a imagem corporal. Assim, parece ser pertinente monitorar a insatisfação corporal, com o intuito de desenvolver ações multidisciplinares que promovam mudanças, e esclarecimentos, quanto ao perfil morfológico adequado à saúde.

É pertinente lembrar que a imagem corporal é uma construção multidimensional, que representa como os indivíduos pensam, sentem e se comportam a respeito de seus atributos físicos. Ela pode ser vista como a relação entre o corpo de uma pessoa e os processos cognitivos como crenças, valores e atitudes individuais ${ }^{36}$. Apensar, a adolescência é caracterizada por transformações biológicas, físicas, psicológicas e sociais. Nesse contexto, o sujeito pode estar monitorando diversos aspectos do seu corpo simultaneamente, os quais podem ou não ser independentes um do outro ${ }^{37}$. Dado esse caráter multidimensional, a avaliação da imagem corporal é complexa, portanto, o presente estudo trata de um recorte, apenas a percepção da imagem corporal, o que 
pode se caracterizar como a principal limitação deste. No entanto, pesquisas que analisam a insatisfação, ou satisfação, com a imagem corporal facilitam a compreensão dos fatos comuns no contexto social em questão ${ }^{37}$.

Ainda pode ser considerado como limitações o instrumento utilizado para a determinação da percepção da imagem corporal (silhuetas corporais), pois os desenhos das silhuetas são bidimensionais o que pode implicar falhas na representação total do corpo, na distribuição da massa de gordura; além disso, o questionário (silhuetas corporais) não teve sua validade testada em jovens brasileiros, no entanto, possui validade para a população adulta brasilei$\mathrm{ra}^{38}$. Por outro lado, é interessante destacar que este foi o primeiro estudo a investigar a associação da insatisfação com a imagem corporal com diferentes indicadores antropométricos em adolescentes rurais e urbanos de um município de pequeno porte do estado de Santa Catarina. Além disso, destaca-se a representatividade de adolescentes investigados na presente casuística.

Os resultados encontrados no presente estudo permitem concluir que mesmo em um município de pequeno porte, a prevalência de insatisfação com a imagem corporal é elevada entre os adolescentes. Enquanto nos rapazes o IMC baixo e a obesidade abdominal apresentaram associação com a insatisfação com a imagem corporal, nas moças, o IMC e $\sum 2 \mathrm{DC}$ elevados estiveram associados, reforçando as evidências de que os rapazes almejam um porte atlético (supostamente maior desenvolvimento muscular, ou pelo menos maior volume corporal), enquanto as moças desejam um corpo esbelto.

\begin{abstract}
Association between body image dissatisfaction and anthropometric indicators in adolescents

The aim of this study was to evaluate the association between body image dissatisfaction, body mass index (BMI), body adiposity and abdominal obesity in adolescents. A total of 637 adolescent boys and girls (11-17 years) participated. Measures of body weight, height, waist circumference, triceps and medial calf skinfold thickness and, body image were collected. BMI, abdominal obesity and body adiposity were used as references in relation to the outcome. The prevalence of body image dissatisfaction was 60\% (boys $=54.3 \%$; girls $=65.2 \%, p<0.05$ ). Boys with a low BMl and abdominal obesity presented a 4.31 and 4.93 times higher probability of body image dissatisfaction, respectively. In girls, the probability of body dissatisfaction was 6.81 and 1.95 times higher among those with a high BMI and high body adiposity, respectively. Low BMI and abdominal obesity were associated with body image dissatisfaction in boys, whereas in girls an association was observed with high BMI and high body adiposity.
\end{abstract}

KEY WORDS: Anthropometry; Body image; Skinfolds; Abdominal fat; BMI.

\title{
Referências
}

1. Ricciardelli LA, McCabe MP. A biopsychosocial model of disordered eating and the pursuit of muscularity in adolescent boys. Psychol Bull. 2004;130:179-205.

2. McCabe MP, Ricciardelli L. Body image dissatisfaction among males across the lifespan: a review of past literature. J Psychosom. 2004;56:675-85.

3. Latzer Y, Tzischinsky O, Asaiza F. Disordered eating related behaviors among Arab schoolgirls in Israel: an epidemiological study. Int J Eat Disord. 2007;40:263-70.

4. Corseuil MW, Pelegrini A, Beck C, Petroski EL. Prevalência de insatisfação com a imagem corporal e sua associação com a inadequação nutricional em adolescentes. Rev Educ Fís/UEM. 2009;20:25-31.

5. Petroski EL, Pelegrini A, Glaner MF. Insatisfação corporal em adolescentes rurais e urbanos. Motricidade. 2009;5:13-25.

6. Pelegrini A, Petroski EL. The association between body dissatisfaction and nutritional status in adolescents. Hum Mov. 2010;11:51-7. 
7. Erickson SJ, Gerstle M. Investigation of ethnic differences in body image between Hispanic/biethnic-Hispanic and non-Hispanic White preadolescent girls. Body Image. 2007;4:69-78.

8. Alves E, Vasconcelos FAG, Calvo MCM, Neves J. Prevalência de sintomas de anorexia nervosa e insatisfação com a imagem corporal em adolescentes do sexo feminino do município de Florianópolis, Santa Catarina, Brasil. Cad Saúde Pública. 2008;24:503-12.

9. Lunardi CC, Petroski EL. Índice de massa corporal, circunferência da cintura e dobra cutânea triciptal na predição de alterações lipídicas em crianças com 11 anos de idade. Arq Bras Endocrinol Metab. 2008;52:1009-14.

10. Rodrigues NA, Perez AJ, Pires JGP, et al. Fatores de risco cardiovasculares, suas associações e presença de síndrome metabólica em adolescentes. J Pediatr. (Rio J.) 2009;85:55-60.

11. Glaner MF, Pelegrini A, Nascimento TBR. Perímetro do abdômen é o melhor indicador antropométrico de riscos para doenças cardiovasculares. Rev Bras Cineantropom Desempenho Hum. 2011;13:1-7.

12. Saudades (Município). Saudades. Saudades: Prefeitura Municipal de Saudades; [citado 11 mar. 2009]. Disponível em: http://www.saudades.sc.gov.br.

13. Programa das Nações Unidas para o Desenvolvimento (PNUD). Ranking do índice de desenvolvimento municipal dos municípios do Brasil. Brasília: PNUD Brasil; [citado 11 mar 2009]. Disponível em: http://www.pnud.org.br.

14. Luiz RR, Magnanini MMF. A lógica da determinação do tamanho da amostra em investigações epidemiológicas. Cad Saúde Colet. 2000;8:9-28.

15. Triches RM, Giugliani ERJ. Insatisfação corporal em escolares de dois municípios da região Sul do Brasil. Rev Nutr. 2007;20:119-28.

16. Stunkard AJ, Sorenson T, Schlusinger F. Use of the Danish adoption register for the study of obesity and thinness. In: Kety SS, Rowland LP, Sidman RL, Matthysse SW, editors. The genetics of neurological and psychiatric disorders. New York: Raven; 1983. p. 115-20.

17. Petroski EL, organizador. Antropometria: técnicas e padronizações. 3a ed. Blumenau: Nova Letra; 2007.

18. Cole TJ, Bellizzi MC, Flegal KM, Dietz WH. Establishing a standard definition for child overweight and obesity worldwide: international survey. Br Med J. 2002;320:1240-3.

19. Cole TJ, Flegal KM, Nicholls D, Jackson AA. Body mass index cut offs to define thinness in children and adolescents: international survey. Br Med J. 2007;335:194.

20. American Alliance for Health, Physical Education, Recreation and Dance (AAHPERD). Physical best. Reston: AAHPERD; 1988.

21. Fernandez JR, Redden DT, Pietrobelli A, Allison DP. Waist circumference percentiles in nationally representative samples of African-American, European-American, and Mexican-American children and adolescents. J Pediatr. 2004;145:439-44.

22. Vilela JEM, Lamounier JA, Dellaretti Filho MA, Barros Neto JR, Horta GM. Transtornos alimentares em escolares. J Pediatr. 2004;80:49-54.

23. Graup S, Pereira EF, Lopes AS, Araújo VC, Legnani RFS, Borgatto, AF. Associação entre a percepção da imagem corporal e indicadores antropométricos de escolares. Rev Bras Educ Fís Esporte. 2008;22:129-38.

24. Vilela JEM, Lamounier JA, Oliveira RG, Ribeiro RQC, Gomes ELC, Barros Neto JR. Avaliação do comportamento alimentar em crianças e adolescentes de Belo Horizonte. Psiquiatr Biol. 2001;9:121-30.

25. Oliveira FP, Bosi MLM, Vigário OS, Vieira RS. Comportamento alimentar e imagem corporal em atletas. Rev Bras Med Esporte. 2003;9:357-64.

26. Jones DC, Vigfusdottir TH, Lee Y. Body Image and the appearance culture among adolescent girls and boys: an examination of friend conversations, peer criticism, appearance magazines, and the internalization of appearance ideals. J Adolesc Res. 2004;19:323-39.

27. Branco LM, Hilário MOE, Cintra IP. Percepção e satisfação corporal em adolescentes e a relação com seu estado nutricional. Rev Psiquiatr Clín. 2006;33:292-6.

28. Glaner MF. Índice de massa corporal como indicador da gordura corporal comparado às dobras cutâneas. Rev Bras Med Esporte. 2005;11:243-6.

29. McCabe MP, Ricciardelli L, Waqa G, Goundar R, Fotu K. Body image and body change strategies among adolescent males and females from Fiji, Tonga and Australia. Body Image. 2009;6:299-303.

30. Pinheiro AP, Giugliani ER. Body dissatisfaction in Brazilian schoolchildren: prevalence and associated factors. Rev Saúde Pública. 2006;40:489-96.

31. Assis SG, Avanci JQ, Silva CMFP, Malaquias JV, Santos NC. Oliveira RVC. A representação social do ser adolescente: um passo decisivo na promoção da saúde. Ciênc Saúde Colet. 2003;8:669-80. 
32. Cortese S, Falissard B, Angriman M, et al. The Relationship between body size and depression symptoms in adolescents. J Pediatr. 2009;154:86-90.

33. Bath JA, Baur LA. Management and prevention of obesity and its complications in children and adolescents. Med J Aust. 2005;182:130-5.

34. Glaner MF. Aptidão física relacionada à saúde de adolescentes rurais e urbanos em relação a critérios de referência. Rev Bras Med Esporte. 2005;19:13-24.

35. Petroski EL, Pelegrini A, Glaner MF. Motivos e prevalência de insatisfação com a imagem corporal em adolescentes. Ciênc Saúde Colet. 2012;17:1071-7.

36. Fischer S. Development and structure of the body image. New Jersey: LEA; 1986.

37. Ribeiro PRL, Tavares MCF. As contribuições de Seymour Fisher para os estudos em imagem corporal. Motricidade. 2011;7:83-95.

38. Scagliusi FB, Alvarenga M, Polacow VO, et al. Concurrent and discriminate validity of the Stunkard's figure rating scale adapted into Portuguese. Appetite. 2006;47:77-82.

\section{Agradecimentos}

Os autores agradecem ao CNPq - Conselho Nacional de Desenvolvimento Científico e Tecnológico, Processo 481859/2007-1, pelo fomento recebido.

Aos alunos, respectivos pais, professores, funcionários e direçōes das EEB Rodrigues Alves e EEB João Batista Fleck, pela distinta colaboração durante a coleta de dados.

\begin{tabular}{r|r} 
ENDEREço & \\
Maria Fátima Glaner & \\
Centro de Educação Física e Esporte & Recebido para publicação: 05/01/2011 \\
Universidade Estadual de Londrina & Revisão: 11/05/2012 \\
Rod. Celso Garcia Cid, PR 445, km 380 & Aceito: 25/05/2012 \\
Campus Universitário - Caixa Postal 6001 & \\
86051-980 - Londrina - PR - BRASIL & \\
e-mail: mfglaner@gmail.com &
\end{tabular}

136 • Rev Bras Educ Fís Esporte, (São Paulo) 2013 Jan-Mar;27(1):129-36 